\title{
Feynman path-integral treatment of the BEC-impurity polaron
}

\author{
J. Tempere, ${ }^{1,2}$ W. Casteels,${ }^{1}$ M. K. Oberthaler, ${ }^{3}$ S. Knoop, ${ }^{3}$ E. Timmermans, ${ }^{4}$ and J. T. Devreese ${ }^{1}$ \\ ${ }^{1}$ TFVS, Universiteit Antwerpen, Groenenborgerlaan 171, B2020 Antwerpen, Belgium \\ ${ }^{2}$ Lyman Laboratory of Physics, Harvard University, Cambridge, Massachusetts 02138, USA \\ ${ }^{3}$ Kirchhoff-Institut für Physik, Universität Heidelberg, Im Neuenheimer Feld 227, D-69120 Heidelberg, Germany \\ ${ }^{4}$ Theoretical Division (T-4), Los Alamos National Laboratory, Los Alamos, New Mexico 87545, USA \\ (Received 2 June 2009; revised manuscript received 19 September 2009; published 10 November 2009)
}

\begin{abstract}
The description of an impurity atom in a Bose-Einstein condensate can be cast in the form of Fröhlich's polaron Hamiltonian, where the Bogoliubov excitations play the role of the phonons. An expression for the corresponding polaronic coupling strength is derived, relating the coupling strength to the scattering lengths, the trap size and the number of Bose condensed atoms. This allows to identify several approaches to reach the strong-coupling limit for the quantum gas polarons, whereas this limit was hitherto experimentally inaccessible in solids. We apply Feynman's path-integral method to calculate for all coupling strengths the polaronic shift in the free energy and the increase in the effective mass. The effect of temperature on these quantities is included in the description. We find similarities to the acoustic polaron results and indications of a transition between free polarons and self-trapped polarons. The prospects, based on the current theory, of investigating the polaron physics with ultracold gases are discussed for lithium atoms in a sodium condensate.
\end{abstract}

DOI: 10.1103/PhysRevB.80.184504

PACS number(s): 74.20.Mn, 71.38.Fp, 03.75.Hh, 67.85.De

\section{INTRODUCTION}

Quantum gases are used as an excellent testbed for manybody theory, and are particularly useful to investigate strongcoupling regimes or strongly correlated regimes that have remained out of reach in the solid state. ${ }^{1}$ The current work focuses on the physics of an impurity in a Bose-Einstein condensate (BEC), as this system opens a promising avenue to investigate long-standing problems in polaron theory. ${ }^{2} \mathrm{In}$ 1952, Fröhlich, inspired by Landau's concept of the polaron, derived a Hamiltonian that describes a charge carrier (electron, hole) interacting with its self-induced polarization, in an ionic crystal or a polar semiconductor. ${ }^{3,4}$ The Fröhlich polaron Hamiltonian has resisted exact analytical diagonalization since 1952 and became a laboratory to test methods of quantum field theory. ${ }^{2}$ Tomonaga's canonical transformation was applied to study the weak-coupling regime. Bogoliubov tackled the polaron strong-coupling limit with one of his canonical transformations. Feynman used his path integral formalism (and an ad hoc variational principle) to develop a superior all coupling approximation for the polaron. ${ }^{5}$ These studies were addressing the self-energy, the effective mass and the mobility of Fröhlich polarons. Studies of the optical absorption of a Fröhlich polaron were initiated by Evrard et al. ${ }^{6}$ for strong coupling and by Gurevich et al. ${ }^{7}$ for the weak-coupling limit. Devreese $e t a l .^{8}$ calculated the optical absorption of the Fröhlich polaron at all coupling using Feynman path integrals and the response formalism introduced in Ref. 9. The results of Ref. 8 reveal the internal excitation structure of the Fröhlich polaron that is absent in Ref. 9.

However the largest Fröhlich polaron coupling constant established in any solid by experiment is not large enough to reveal the rich internal excitation structure of the optical absorption predicted in Ref. 8. The possibility to tune the coupling strength in the BEC-impurity system presents a marvelous challenge and opportunity to experimentally reveal the internal excitation structure and its related resonances and scattering states "contained" in the Fröhlich fieldtheoretical polaron Hamiltonian. Quantum gases could therefore reveal important and subtle characteristics (eigenstates, resonances,...) of a Hamiltonian devised for a solid, however, that cannot be realized in a solid. The observation of the spectra of the BEC-impurity Hamiltonian at large coupling would be all the more interesting in view of recent studies of the spectrum of the Fröhlich polaron Hamiltonian with diagrammatic quantum Monte Carlo numerical techniques ${ }^{10}$ and with a strong coupling model ${ }^{11}$ that explore the validity of the Franck-Condon principle with increasing coupling strength. Similarly, the transition between quasifree polarons and self-trapped polarons predicted for acoustic polarons ${ }^{13}$ is expected to occur in a coupling regime hitherto inaccessible in the solid state. Finally, a better understanding of the intermediate and strong-coupling regimes is needed to further elucidate the role of polarons and bipolarons in unconventional pairing mechanisms for high-temperature superconductivity. ${ }^{14}$

The Hamiltonian of an impurity in a Bose-Einstein condensate can be mapped onto the polaron Hamiltonian when the Bogoliubov approximation is valid. ${ }^{15,16}$ The polaronic effects comes about through the coupling of the impurity with the Bogoliubov excitations, as shown in Sec. II. We apply the path-integral formalism, valid at all values of the coupling strength, to describe the polaronic effect in a condensate in Sec. III. As a concrete example, we use the parameters for a lithium impurity in a sodium condensate, and derive an expression linking the experimental parameters to a dimensionless coupling constant analogous to the Fröhlich coupling constant for electrons in polar crystals. The polaronic energy shift, the effective mass increase and the polaron radius are studied for all values of this coupling strength and at all temperatures where the Bogoliubov approximation is valid, in Sec. IV. 


\section{POLARON HAMILTONIAN FOR AN IMPURITY IN A CONDENSATE}

The Hamiltonian of a single atom, in the presence of a Bose gas is given by:

$$
\begin{aligned}
\hat{H}= & \frac{\hat{p}^{2}}{2 m_{I}}+\sum_{\mathbf{k}} \varepsilon_{\mathbf{k}} \hat{a}_{\mathbf{k}}^{\dagger} \hat{a}_{\mathbf{k}}+\frac{1}{2} \sum_{\mathbf{k}, \mathbf{k}^{\prime}, \mathbf{q}} V_{B B}(\mathbf{q}) \hat{a}_{\mathbf{k}^{\prime}-\mathbf{q}}^{\dagger} \hat{a}_{\mathbf{k}+\mathbf{q}}^{\dagger} \hat{a}_{\mathbf{k}} \hat{a}_{\mathbf{k}^{\prime}} \\
& +\sum_{\mathbf{k}, \mathbf{q}} V_{I B}(\mathbf{q}) \hat{\rho}_{I}(\mathbf{q}) \hat{a}_{\mathbf{k}^{\prime}-\mathbf{q}}^{\dagger} \hat{a}_{\mathbf{k}^{\prime}}
\end{aligned}
$$

The first term represents the kinetic energy of the "impurity" atom with mass $m_{I}$. The operators $\hat{a}_{\mathbf{k}}^{\dagger}, \hat{a}_{\mathbf{k}}$ create and annihilate a boson with mass $m_{B}$, wave number $\mathbf{k}$, and energy $\varepsilon_{\mathbf{k}}$ $=(\hbar k)^{2} /\left(2 m_{B}\right)-\mu$ where $\mu$ is the chemical potential. These bosons interact, and $V_{B B}(\mathbf{q})$ is the Fourier transform of the boson-boson interaction potential. The interaction between the bosonic atoms and the impurity atom is described by a potential $V_{I B}(\mathbf{q})$ coupling the boson density to the impurity density $\hat{\rho}_{I}(q)$, which can be expressed as a function of the impurity position operator $\hat{r}$ as

$$
\hat{\rho}_{I}(\mathbf{q})=\int d^{3} \mathbf{r} e^{-i \mathbf{q} \cdot \mathbf{r}} \delta(\mathbf{r}-\hat{\mathbf{r}}) .
$$

Bose-Einstein condensation is realized when the singleparticle density matrix has an eigenvalue $N_{0} \gg 1$ comparable to the total number of bosons. ${ }^{19}$ For a homogeneous condensate, this is expressed by the Bogoliubov shift, ${ }^{20}$ which transforms the Hamiltonian (1) into

$$
\begin{aligned}
\hat{H}= & E_{G P}+N_{0} V_{I B}(\mathbf{0})+\frac{\hat{p}^{2}}{2 m_{I}}+\sum_{\mathbf{k} \neq 0} E_{\mathbf{k}} \hat{b}_{\mathbf{k}}^{\dagger} \hat{b}_{\mathbf{k}} \\
& +\sum_{\mathbf{k} \neq 0} \sqrt{\frac{\xi_{\mathbf{k}} N_{0}}{E_{\mathbf{k}}}} V_{I B}(\mathbf{k}) \hat{\rho}_{I}(\mathbf{k})\left[\hat{b}_{\mathbf{k}}+\hat{b}_{-\mathbf{k}}^{\dagger}\right] .
\end{aligned}
$$

Here, the operators $\hat{b}_{\mathbf{k}}^{\dagger}, \hat{b}_{\mathbf{k}}$ create, respectively, annihilate Bogoliubov excitations with wave number $\mathbf{k}$ and dispersion

$$
E_{\mathbf{k}}=\sqrt{\xi_{\mathbf{k}}\left[\xi_{\mathbf{k}}+2 N_{0} V_{B B}(\mathbf{k})\right]}
$$

where $\xi_{\mathbf{k}}=(\hbar k)^{2} /\left(2 m_{B}\right)$. The first term in Eq. (3) represents the Gross-Pitaevskii energy ${ }^{21}$

$$
E_{G P}=N_{0} \varepsilon_{0}+\frac{N_{0}^{2}}{2} V_{B B}(\mathbf{0})+\frac{1}{2} \sum_{\mathbf{k} \neq 0} N_{0} V_{B B}(\mathbf{k}),
$$

and the second term is the interaction shift due to the impurity. For both the boson-boson interaction and the bosonimpurity interaction we will assume contact pseudopotentials: $V_{B B}\left(\mathbf{r}-\mathbf{r}^{\prime}\right)=g_{B B} \delta\left(\mathbf{r}-\mathbf{r}^{\prime}\right)$ and $V_{I B}\left(\mathbf{r}-\mathbf{r}^{\prime}\right)=g_{I B} \delta\left(\mathbf{r}-\mathbf{r}^{\prime}\right)$. The interaction strengths $g_{B B}$ and $g_{I B}$ are related to the boson-boson scattering length $a_{B B}$ and the boson-impurity scattering length $a_{I B}$, respectively, through the LippmannSchwinger equation. For the boson-boson interaction, the first-order result $g_{B B}=4 \pi \hbar^{2} a_{B B} / m_{B}$ will suffice. For the boson-impurity interaction, the Lippmann-Schwinger equation needs to be treated correctly up to second order to obtain valid results for the polaron problem, since as we shall see, $g_{I B}^{2}$ will appear in the expressions.

The resulting Hamiltonian (3) maps onto the Fröhlich polaron Hamiltonian 4

$$
\hat{H}_{p o l}=\frac{\hat{p}^{2}}{2 m_{I}}+\sum_{\mathbf{k} \neq 0} \hbar \omega_{\mathbf{k}} \hat{b}_{\mathbf{k}}^{\dagger} \hat{b}_{\mathbf{k}}+\sum_{\mathbf{k} \neq 0} V_{\mathbf{k}} e^{i \mathbf{k} \cdot \hat{\mathbf{r}}}\left(\hat{b}_{\mathbf{k}}+\hat{b}_{-\mathbf{k}}^{\dagger}\right)
$$

with

$$
\begin{gathered}
\hbar \omega_{\mathbf{k}}=c k \sqrt{1+(\xi k)^{2} / 2} \\
V_{\mathbf{k}}=\sqrt{N_{0}}\left[\frac{(\xi k)^{2}}{(\xi k)^{2}+2}\right]^{1 / 4} g_{I B} .
\end{gathered}
$$

In these expressions $\xi=1 / \sqrt{8 \pi a_{B B} n_{0}}$ is the healing length of the Bose condensate with $n_{0}=N_{0} / V$ the condensate density (in the present calculations for the homogeneous gas we work with unit volume) and $c=\hbar /\left(\sqrt{2} m_{B} \xi\right)$ is the speed of sound in the condensate. The operator structure of the BECimpurity Hamiltonian and that of the Fröhlich polaron Hamiltonian is identical. Depending on the analytical form of the scalar functions multiplying the field operators, the Fröhlich polaron Hamiltonian, originally devised to describe the electron/hole-longitudinal optical phonon interaction, can depict as well the acoustopolaron, ${ }^{12,13}$ the piezopolaron, ${ }^{17}$ the ripplopolaron, ${ }^{18}$, etc. However, a formalism/method that diagonalizes the Fröhlich Hamiltonian at all coupling, does so for all those different types of polarons, including the BEC-impurity polaron.

This mapping of the BEC-impurity problem onto the Fröhlich Hamiltonian, and the resulting possibility of polaronic self-trapping of an impurity in a condensate, has been the subject of recent theoretical investigations at strong coupling, ${ }^{15,16}$ and at low coupling with the Lee-Low-Pines scheme. ${ }^{22}$ For small polarons in an optical lattice, a perturbative study exists. ${ }^{23}$ As is also known from the study of the polaronic problem for slow electrons in a polar crystal, these approximations give qualitatively different results for different regimes of coupling strength. These different characteristics between weak-coupling and strong-coupling behavior are even more pronounced in the case of an impurity in a condensate. In this work, we present a calculation valid for all coupling strengths, based on the Jensen-Feynman variational scheme first successfully applied by Feynman ${ }^{5}$ to study slow electrons interacting with optical phonons in polar crystals. As discussed below, the present system will have more similarities to acoustic polarons than to the Fröhlich polarons.

\section{JENSEN-FEYNMAN FREE ENERGY}

\section{A. General treatment}

The calculation of the free energy $F$ of the polaron is based on the Jensen-Feynman variational inequality ${ }^{24,25}$

$$
F \leq F_{0}+\frac{1}{\hbar \beta}\left\langle\mathcal{S}-\mathcal{S}_{0}\right\rangle_{\mathcal{S}_{0}}
$$

where $\mathcal{S}$ is the action functional of the real system described by the Hamiltonian (6), and $\mathcal{S}_{0}$ is the action functional 
of a variational model system with free energy $F_{0}$, and $\beta=1 /\left(k_{B} T\right)$ with $T$ the temperature. This variational principle is an extension of the usual Gibbs-Bogoliubov variational principle $F \leq F_{0}+\left\langle\hat{H}-\hat{H}_{0}\right\rangle$, where $\hat{H}$ is the Hamiltonian of the system under study and $\hat{H}_{0}$ is a model Hamiltonian, and the brackets indicate statistical averaging. The inequality (9) can be derived ${ }^{24,25}$ from the path-integral expression for the partition sum:

$$
\begin{aligned}
e^{-\beta F} & =\int \mathcal{D} \mathbf{r} e^{-\mathcal{S}[\mathbf{r}(t)] / \hbar}=\int \mathcal{D} \mathbf{r} e^{-\left\{\mathcal{S}[\mathbf{r}(t)]-\mathcal{S}_{0}[\mathbf{r}(t)]\right\} / \hbar} e^{-\mathcal{S}_{0}[\mathbf{r}(t)] / \hbar} \\
& =e^{-\beta F_{0}}\left\langle e^{-\left(\mathcal{S}-\mathcal{S}_{0}\right) / \hbar}\right\rangle_{\mathcal{S}_{0}} \geq \exp \left[-\beta F_{0}-\left\langle\mathcal{S}-\mathcal{S}_{0}\right\rangle_{\mathcal{S}_{0}} / \hbar\right]
\end{aligned}
$$

where the inequality follows from the convexity of the exponential, and $\mathcal{D} \mathbf{r}$ represents the path-integral measure. The peculiarity of this variational principle, in comparison to the usual Rayleigh-Ritz variational approach, is that a variational action functional is used rather than a variational wave function. One important strength of the Jensen-Feynman principle is that it can be used at nonzero temperatures. ${ }^{24}$ Another strength, in comparison with the Gibbs-Bogoliubov variational principle, is that it can treat systems that are hard or impossible to describe in the standard Hamiltonian formalism, such as a collection of particles that interact through a retarded potential, such as Lienard-Wiechert potentials in electrodynamics, or the phonon-induced electron-electron potential in polaron theory. In particular, the polaron Hamiltonian (6) after the elimination of the phonon degrees of freedom leads to an action functional $\mathcal{S}$ containing retardation effects:

$$
\begin{aligned}
\mathcal{S}= & \int_{0}^{\hbar \beta} \frac{m_{I}}{2} \dot{r}^{2}(\tau) d \tau-\sum_{\mathbf{k} \neq 0} \frac{\left|V_{\mathbf{k}}\right|^{2}}{2 \hbar} \int_{0}^{\hbar \beta} d \tau \int_{0}^{\hbar \beta} d \sigma \\
& \times \mathcal{G}(\mathbf{k},|\tau-\sigma|) e^{i \mathbf{k} \cdot[\mathbf{r}(\tau)-\mathbf{r}(\sigma)]},
\end{aligned}
$$

with $\mathcal{G}(\mathbf{k}, u)$ the Bogoliubov excitation Green's function, given by

$$
\mathcal{G}(\mathbf{k}, u)=\frac{\cosh \left[\omega_{\mathbf{k}}(u-\hbar \beta / 2)\right]}{\sinh \left(\hbar \beta \omega_{\mathbf{k}} / 2\right)} .
$$

The action functional (11) is obtained by integrating out analytically the oscillator degrees of freedom corresponding to the Bogoliubov excitations, a technique introduced in the context of phonons in Ref. 5. This gives rise to a retarded interaction (proportional to $e^{i \mathbf{k} \cdot[\mathbf{r}(\tau)-\mathbf{r}(\sigma)]}$ ), mediated by the Bogoliubov-excitation Green's function, and with coupling strength $\left|V_{\mathbf{k}}\right|^{2} /(2 \hbar)$.

The system under study here is modeled by a variational trial system with free energy $F_{0}$ described by the action functional

$$
\begin{aligned}
\mathcal{S}_{0}= & \int_{0}^{\hbar \beta} \frac{m_{I}}{2} \dot{r}^{2}(\tau) d \tau+\frac{M W^{3}}{8} \int_{0}^{\hbar \beta} d \tau \\
& \times \int_{0}^{\hbar \beta} d \sigma \frac{\cosh [W(|\tau-\sigma|-\hbar \beta / 2)]}{\sinh (\beta \hbar W / 2)}[\mathbf{r}(\tau)-\mathbf{r}(\sigma)]^{2} .
\end{aligned}
$$

The model system corresponds to a mass $m_{I}$ that is coupled by a spring with spring constant $M W^{2}$ to a "Bogoliubov" mass $M$. Both $M$ and $W$ are variational parameters. The expectation value in the inequality is evaluated in the model system. This model system is chosen partly because the expectation values can be calculated analytically for it. For more details on the formalism, we refer to Refs. 5, 24, and 26:

$$
\begin{aligned}
F \leq & \frac{3}{\beta}\left\{\ln \left[2 \sinh \left(\frac{\beta \hbar \Omega}{2}\right)\right]-\ln \left[2 \sinh \left(\frac{\beta \hbar W}{2}\right)\right]\right\} \\
& -\frac{3}{2 \beta} \frac{M}{m_{I}+M}\left[\frac{\hbar \beta \Omega}{2} \operatorname{coth}\left(\frac{\beta \hbar \Omega}{2}\right)-1\right] \\
& -\sum_{\mathbf{k}} \frac{\left|V_{\mathbf{k}}\right|^{2}}{\hbar} \int_{0}^{\hbar \beta} d u\left(1-\frac{u}{\hbar \beta}\right) \mathcal{G}(\mathbf{k}, u) \mathcal{M}_{M, \Omega}(\mathbf{k}, u) .
\end{aligned}
$$

Here $\Omega=W \sqrt{1+M / m_{I}}$, and the memory function is given by

$$
\begin{aligned}
\mathcal{M}_{M, \Omega}(\mathbf{k}, u)= & \exp \left[-\frac{\hbar k^{2}}{2\left(m_{I}+M\right)}\left(u-\frac{u^{2}}{\hbar \beta}\right.\right. \\
& \left.\left.-\frac{M}{m_{I}} \frac{\cosh [\Omega(\hbar \beta / 2)]-\cosh [\Omega(\hbar \beta / 2-u)]}{\Omega \sinh (\beta \hbar \Omega / 2)}\right)\right] .
\end{aligned}
$$

Rather than choosing $W, M$ as variational parameters, we can choose $\Omega, M$ as variational parameters.

\section{B. BEC-impurity}

Care should be taken when we substitute the interaction amplitude (8) into these expressions. Since $\left|V_{\mathbf{k}}\right|^{2} \propto g_{I B}^{2}$, we need to solve the Lippmann-Schwinger equation up to second order to obtain the link between $g_{I B}$ and $a_{I B}$ correctly; we find

$$
\frac{2 \pi \hbar^{2} a_{I B}}{m_{r}}=g_{I B}-g_{I B}^{2} \sum_{\mathbf{k}^{\prime} \neq 0} \frac{2 m_{r}}{\left(\hbar k^{\prime}\right)^{2}},
$$

where $m_{r}$ is the relative mass $\left(m_{r}^{-1}=m_{B}^{-1}+m_{I}^{-1}\right)$. From this, we find that the term $N_{0} V_{I B}(\mathbf{0})=N_{0} g_{I B}$ in Eq. (3) must also contribute a renormalization factor since

$$
N_{0} g_{I B} \rightarrow N_{0}\left(\frac{2 \pi \hbar^{2} a_{I B}}{m_{r}}+g_{I B}^{2} \sum_{\mathbf{k}^{\prime} \neq 0} \frac{2 m_{r}}{\left(\hbar k^{\prime}\right)^{2}}\right)
$$

Substituting Eqs. (8) and (7) into Eq. (14) then yields 


$$
\begin{aligned}
F \leq & \frac{3}{\beta}\left\{\ln \left[2 \sinh \left(\frac{\beta \Omega}{2}\right)\right]-\ln \left[2 \sinh \left(\frac{\beta \Omega}{2 \sqrt{1+M}}\right)\right]\right\} \\
& -\frac{3}{2 \beta} \frac{M}{1+M}\left[\frac{\beta \Omega}{2} \operatorname{coth}\left(\frac{\beta \Omega}{2}\right)-1\right] \\
& +\alpha\left(\frac{\tilde{m}_{B}+1}{\tilde{m}_{B}}\right)^{2} \int_{0}^{\infty} \frac{d k}{4 \pi}\left[\left(\frac{2 \tilde{m}_{B}}{1+\tilde{m}_{B}}\right)\right. \\
& \left.-\frac{k^{3}}{\sqrt{2+k^{2}}} \int_{0}^{\beta / 2} \mathcal{G}(k, u) \mathcal{M}(k, u) d u\right] .
\end{aligned}
$$

In this expression,

$$
\alpha=a_{I B}^{2} /\left(a_{B B} \xi\right)
$$

is the polaronic coupling strength. We use polaronic units, so that $\hbar=m_{I}=\xi=1$. That means the free energy is in units $\hbar^{2} /\left(m_{I} \xi^{2}\right)$, so that $\beta=\hbar^{2} /\left(m_{I} \xi^{2} k_{B} T\right)$ and $\tilde{m}_{B}=m_{B} / m_{I}$. The integration variables $k, u$ and the variational parameters $M, \Omega$ in Eq. (18) are dimensionless. In these units, we find after substitution of Eqs. (8) and (7), that the Green's function is given by

$$
\mathcal{G}(k, u)=\frac{\cosh \left[k \sqrt{k^{2}+2}(2 u-\beta) /\left(4 \tilde{m}_{B}\right)\right]}{\sinh \left[\beta k \sqrt{k^{2}+2} /\left(4 \tilde{m}_{B}\right)\right]},
$$

and the memory function takes the form

$$
\begin{aligned}
\mathcal{M}(k, u)= & \exp \left[-\frac{k^{2}}{2(1+M)}\left(\frac{u(\beta-u)}{\beta}\right.\right. \\
& \left.\left.+M \frac{\cosh [\Omega(\beta / 2)]-\cosh [\Omega(\beta / 2-u)]}{\Omega \sinh (\beta \Omega / 2)}\right)\right] .
\end{aligned}
$$

The first term of the integrand of the $k$-integral in Eq. (18) is due to a renormalization factor, which arises when we relate $g_{I B}$ to the boson-impurity scattering length. This term is independent of the variational parameters $M$ and $\Omega$ and does not influence the optimization of the variational parameters; it is however necessary for the $k$-integrals to converge. In the case of the acoustic polaron, convergence in the variational minimization of Eq. (18) is sped up by introducing a cutoff $K_{c}$ to the wave-number integral, ${ }^{12,13}$ for which there is a natural choice, namely, the edge of the Brillouin zone. For atomic gases, as we show in Sec. IV, the results do not strongly depend on the value cutoff as long as it is on the order of the (inverse) Van der Waals radius which we use as a natural scale for $K_{c}$ in the present case. Note that, although these formulas are derived for any temperature (any $\beta$ ), the mapping onto the polaron Hamiltonian is only valid for temperatures below the critical temperature, and low enough so that the number of Bogoliubov excitations can be neglected with respect to the number of atoms in the condensate, so that Eq. (3) gives a good description of the quantum gas. In the discussion, we will look at realistic experimental values for $\beta$ and other parameters.

\section{Weak and strong coupling limits}

From our central result [Eq. (18)], we can retrieve both the weak-coupling and the strong-coupling results by a judicious choice of variational parameters, or rather variational trial actions. The weak-coupling limit is obtained by taking the limit $M \rightarrow 0$ in the Jensen-Feynman treatment, which corresponds to a the trial action

$$
\mathcal{S}_{0}^{\text {weak }}=\int_{0}^{\hbar \beta} \frac{m_{I}}{2} \dot{r}^{2}(\tau) d \tau
$$

of a free particle, as pointed out in the original work by Feynman. ${ }^{5}$ In the limit of zero temperature, this corresponds exactly to the second-order perturbation result $E^{\text {weak }}(\alpha)$; we find

$$
\begin{aligned}
E^{\text {weak }}= & \alpha \frac{\left(1+\tilde{m}_{B}\right)^{2}}{\tilde{m}_{B}} \frac{1}{2 \pi} \int_{0}^{\infty} d q\left[\frac{1}{1+\tilde{m}_{B}}\right. \\
& \left.-\sqrt{\frac{q^{2}}{q^{2}+2}} \frac{q}{\tilde{m}_{B} q+\sqrt{q^{2}+2}}\right] .
\end{aligned}
$$

Note that the variational energy becomes independent of $\Omega$ in the limit $M \rightarrow 0$. The energy grows linearly with $\alpha$, and the proportionality constant depends on $m_{B}$ :

$$
E_{\mathrm{pol}}^{\mathrm{weak}}(\alpha)=\left\{\begin{array}{ll}
\alpha /(\sqrt{2} \pi) & \text { for } m_{B} \rightarrow \infty \\
4 \sqrt{2} \alpha /(3 \pi) & \text { for } m_{B} \rightarrow 1 \\
\alpha /\left(\sqrt{2} m_{B}\right) & \text { for } m_{B} \rightarrow 0
\end{array} .\right.
$$

The strong-coupling limit is derived by taking the limit $M$ $\rightarrow \infty$ in the Jensen-Feynman treatment. In this limit, the variational particle with mass $M \rightarrow \infty$ is fixed in place, and we get a particle attached by a spring to a fixed point rather than to a mobile variational mass. The resulting trial action for strong coupling is 5

$$
\mathcal{S}_{0}^{\text {strong }}=\int_{0}^{\hbar \beta}\left(\frac{m_{I}}{2} \dot{r}^{2}(\tau)+\frac{m_{I} \Omega^{2}}{2} r^{2}(\tau)\right) d \tau
$$

relating the strong-coupling limit to the variational approach of Landau and Pekar $^{27}$ for the polaron problem, based on a Gaussian variational wave function. The variational energy becomes

$$
\begin{aligned}
E^{\text {strong }} \leq & \frac{3}{4} \Omega+\lim _{\beta \rightarrow \infty} \alpha\left(\frac{\tilde{m}_{B}+1}{\tilde{m}_{B}}\right)^{2} \int_{0}^{\infty} \frac{d k}{4 \pi}\left[\left(\frac{2 \tilde{m}_{B}}{1+\tilde{m}_{B}}\right)\right. \\
& \left.-\frac{k^{3}}{\sqrt{2+k^{2}}} \int_{0}^{\beta / 2} \mathcal{G}(k, u) \mathcal{M}(k, u) d u\right] .
\end{aligned}
$$

Care must be taken with the limit $\beta \rightarrow \infty$ : since the convergence of $\mathcal{G}(k, u)$ to its $\beta \rightarrow \infty$ limit is not uniform the limit cannot be interchanged with the integration.

\section{RESULTS AND DISCUSSION}

\section{A. Experimental realization}

Before we turn to the results, it is useful to find typical experimental values of the system parameters. Consider a 
sodium condensate of $N_{0}=10^{5}$ atoms in a harmonic trap with axial and radial trapping frequencies $\omega_{\mathrm{ax}}=2 \pi \cdot 50 \mathrm{~Hz}$ and $\omega_{\text {rad }}=2 \pi \cdot 150 \mathrm{~Hz}$, respectively. As scattering length for sodium in the $\left|F, M_{F}\right\rangle=|1,1\rangle$ hyperfine state, we use $a_{B B}$ $=2.8 \mathrm{~nm} .{ }^{28}$ The condensate is suitably described by the Thomas-Fermi (TF) approximation. In our calculations we have assumed spatial homogeneity. The density appears in the theoretical results through the healing length, which is the relevant length scale for the polaronic effect, and appears in expression (19) for the coupling constant. As long as the polaronic effects are restricted to a range considerably smaller than the TF radius $(9.5 \mu \mathrm{m})$, we can use the local density at the center of the trap, $n_{0}=7 \times 10^{13} \mathrm{~cm}^{-3}$, to estimate the appropriate healing length. In the current example, it is $\xi=450 \mathrm{~nm}$ at the center of the trap, indeed smaller than the TF radius. Using $\xi=1 / \sqrt{8 \pi n_{0} a_{B B}}$ and the Thomas-Fermi expression for $n_{0}$ in the expression (19), we find the useful relation for the coupling constant

$$
\alpha=15^{1 / 5}\left(\frac{N_{0} a_{B B}}{\bar{a}_{H O}}\right)^{1 / 5} \frac{a_{I B}^{2}}{a_{B B} \bar{a}_{H O}},
$$

expressing the polaronic coupling constant as a function of the number of condensate atoms $N_{0}$, the oscillator length $\bar{a}_{H O}=\sqrt{\hbar /\left(m \omega_{H O}\right)}$, associated with the geometrical average trap frequency $\omega_{H O}=\sqrt[3]{\omega_{\mathrm{ax}} \omega_{\mathrm{rad}}^{2}}$, and the $s$-wave scattering lengths $a_{B B}$ and $a_{I B}$.

For the impurity, we consider a ${ }^{6} \mathrm{Li}$ atom, so that

$$
\tilde{m}_{B}=m_{B} / m_{I}=3.8 \text {. }
$$

Using $a_{I B}=0.8 \mathrm{~nm}$ as the ${ }^{6} \mathrm{Li}^{-23} \mathrm{Na}$ scattering length, ${ }^{29} \alpha$ is of the order of $10^{-3}$. For these values, the weak-coupling expression (22) is suitable. To increase $\alpha$, several strategies are possible. One possibility is to increase $a_{I B} / a_{B B}$ using Feshbach resonances to either increase $a_{I B}$ or decrease $a_{B B}$. One could also increase $a_{I B} / \bar{a}_{H O}$ by tightening the trap. The remaining factor in expression (25) varies slowly as a function of the TF parameter $N_{0} a_{B B} / \bar{a}_{H O}$.

We look in particular at two strategies for increasing $\alpha$ in the case of lithium impurities in a sodium condensate. On the one hand, one can minimize $a_{B B}$ using the zero-crossing near the sodium Feshbach resonance at 907 G. ${ }^{30}$ The tunability of $a_{B B}$ is limited by the magnetic field stability; we expect a factor of 10 increase in $\alpha$ to be realistic. On the other hand, one can increase $a_{I B}$ using a sodium-lithium Feshbach resonance, for which the prime candidates are the already observed resonance at $796 \mathrm{G}^{31}$ or the predicted resonance at $1186 \mathrm{G}^{29}$ In this strategy, the limiting factor to the tunability of $a_{I B}$ is the trapping lifetime of the lithium atoms. The main loss process is three-body recombination, involving one lithium and two sodium atoms, which is strongly enhanced near the lithium-sodium resonance as the loss scales as $a_{I B}^{4}$. We expect that an increase in $\alpha\left(\propto a_{I B}^{2}\right)$ up to a factor 1000 are still attainable. This leads to a maximal $\alpha$ of order unity, which is in the crossover to the strong-coupling regime. In addition, to avoid phase separation or collapse the trapping potential and the number of lithium atoms have to be carefully chosen.

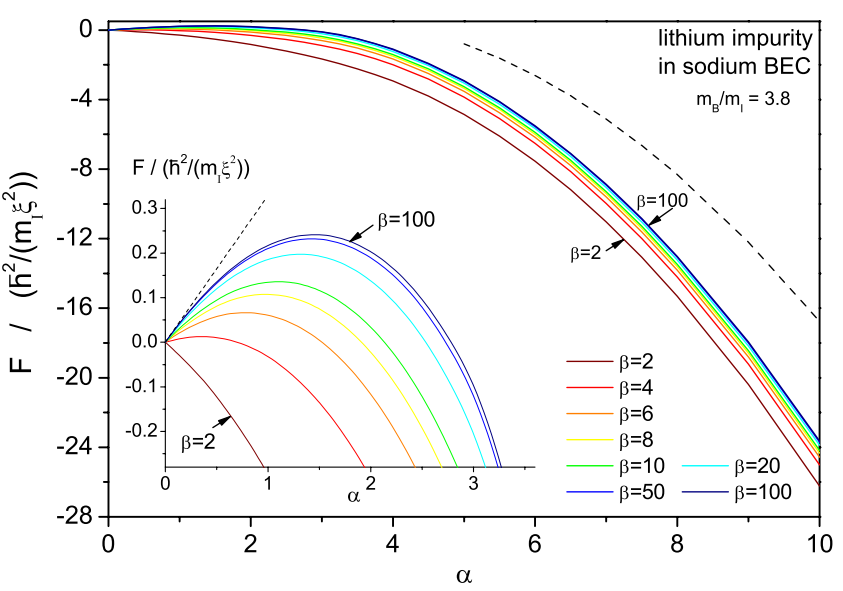

FIG. 1. (Color online) The polaronic contribution Eq. (18) to the free energy of an impurity in a condensate is shown as a function of the coupling constant $\alpha$, for different values of the temperature. From top to bottom, these are $\beta=100,50,20,10,8,6,4$, and 2, corresponding in our example to $T=3.5,7,18,35,44,58,88$, and $175 \mathrm{nK}$, respectively. The dashed curve shows the strong-coupling Pekar approximation. The inset zooms in on the small $\alpha$ region: the dashed line in inset shows the second order perturbation result.

The energy scale $\hbar^{2} /\left(m_{I} \xi^{2}\right)$ corresponds to a frequency of $2 \pi \cdot 8.1 \mathrm{kHz}$ or a temperature of ca. $390 \mathrm{nK}$. With this choice,

$$
\beta=\frac{390 \mathrm{nK}}{T(\mathrm{nK})} .
$$

The (ideal gas) critical temperature for the sodium condensate is $220 \mathrm{nK}$, or $\beta=1.8$. As the lowest temperature we estimate $0.5 T_{\mu}$ where $T_{\mu}=\mu / k_{B}=53 \mathrm{nK}$. This corresponds to $\beta=15$, so that the experimentally relevant window for this parameter is $\beta \in[1.8,15]$.

The Bogoliubov dispersion at low $k$ is similar to that of acoustic phonons rather than to that of optical phonons. For the acoustic polaron, it is known that the variational parameters-especially the polaron mass-depend on the value of a cutoff in the $K_{c}$ integral. ${ }^{13}$ For phonons in solids, this cutoff is related to the edge of the Brillouin zone. In the case of dilute quantum gases, a natural cutoff scale arises from the range $r_{0}$ of the interatomic potential: on scales smaller than this the interaction amplitude cannot be represented any more by expression (8) and should be suppressed. The range of the interatomic potential for sodium is estimated through the Van der Waals radius $r_{0}=2.4 \mathrm{~nm}$, related by $r_{0}=\frac{1}{2}\left(m C_{6} / \hbar^{2}\right)^{1 / 4}$ to the Van der Waals coefficient $C_{6}$ $=1556 .{ }^{32}$ In units of $\xi^{-1}$, this places the cutoff at $K_{c} \approx 200$ for the parameters listed in this section.

\section{B. Free energy and critical coupling strength}

Figure 1 shows the results for the free energy as a function of $\alpha$, using $m_{B} / m_{I}=3.8$ and a wave number cutoff at $K_{c}=200$, for different values of $\beta$, ranging from $\beta=100$ to $\beta=2$, where thermal depletion of the condensate starts to be appreciable. Standard cooling schemes, as mentioned, can cool down to roughly $\beta=15$. The dashed line shows the 


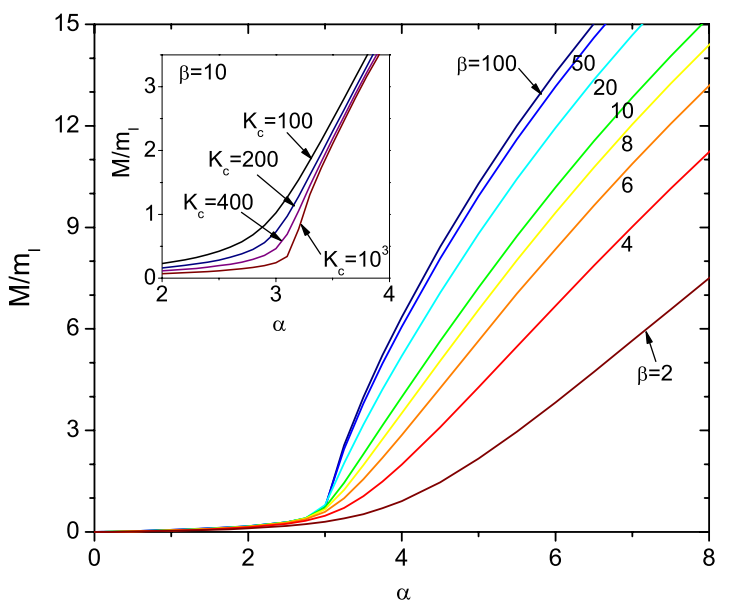

FIG. 2. (Color online) The variational mass parameter $M$ is shown as a function of $\alpha$. The effective polaron mass is to a good approximation given by $m_{p o l}=m_{I}\left(1+M / m_{I}\right)$. The curves correspond to different temperatures $(\beta=100,50,20,10,8,6,4$, and 2 , from top to bottom), at cutoff $K_{c}=200$. The inset shows the effect of cutoff $\left(K_{c}=100,200,400\right.$, and 1000, from top to bottom), at $\beta$ $=10$.

weak-coupling perturbative result valid for temperature zero (and without any wave number cutoff). As predicted by Timmermans and coworkers, at small $\alpha \ll 1$ and $\beta \gg 1$ the polaronic contribution to the energy is positive. It reaches a maximum around $\alpha=1.5-2.0$ and then decreases. The polaronic energy contribution becomes negative (indicative of a transition from an unbound state to a self-trapped state) at a critical coupling strength $\alpha_{c} \simeq 3$ for $T \rightarrow 0$. This critical value goes to zero for increasing temperatures. The perturbative solution is seen to fit well at low coupling. At larger coupling, the dashed curve indicates the strong-coupling variational result [Eq. (24)]: it is significantly larger than the result of the full variation with $M$ and $\Omega$ free parameters, indicating that a Gaussian wave function may not be as suitable as it is for Fröhlich polarons in the solid state. We emphasize that the polaronic energy calculated here is the contribution from Eq. (6) and does not contain the terms $E_{G P}$ $+N_{0} V_{I B}(\mathbf{0})$, which have a known dependence on the various tunable parameters $N_{0}, a_{I B}$ and $a_{B B}$, and which complicate the experimental determination of the polaronic energy contribution. To observe polaronic effects, it may be more straightforward to measure the shift in effective mass of the impurity.

\section{Effective mass increase}

The effective polaron mass $m_{\text {pol }}$ can be derived from the path-integral propagation of a particle from $r(0)$ to a nearby point $r(\tau)=r(0)+v \tau$ by casting the resulting transition amplitude in the form $\exp \left\{-m_{\text {pol }} v^{2} \tau / 2\right\}$. Feynman notes that this procedure gives a value for $m_{\text {pol }}$, which is always within a few percent of $1+M$ with $M$ the variationally optimal mass $M$ of the trial model. ${ }^{24,26}$ Figure 2 shows the result for $M$ as a function of $\alpha$, for different values of the temperature. For small values of the coupling strength, the mass increases linearly with $\alpha$ as predicted by perturbation theory. However, near $\alpha \approx 3.5$, the behavior changes and the mass increases rapidly.

The low-energy Bogoliubov excitations are sound waves, with a dispersion qualitatively similar to acoustic phonons. The effective mass of acoustic polarons-electrons interacting with acoustic phonons-shows a jump of several orders of magnitude in the effective mass $\left(\mathrm{cf}^{13}\right)$ above a critical coupling strength. In the present case we also note a faster increase in the mass above a critical coupling strength, even though the interaction amplitude is different from that between acoustic phonons and electrons. In the dilute atomic gas, the transition is much less dramatic, and becomes smoother as temperature increases. We believe the smoothness of the crossover is not an artifact of the path-integral formalism, since in the case of the acoustic polarons it is the same formalism that predicts a discontinuous jump. For acoustic polarons, it is also predicted that the sharpness of the transition depends strongly on the cutoff: above a critical value of $K_{c}$ a discontinuity appears in the mass as a function of $\alpha$. So it is worthwhile to study the dependence of $M$ on the value of a cut-off to the $k$ integrations in Eq. (18) also in the present case. We find that increasing the cutoff sharpens the transition (as can be seen in the inset of Fig. 2), but no discontinuity arises as it does for the acoustic polaron. The difference is due to the fact that although the Bogoliubov excitation dispersion and the interaction amplitude show a similar $k$-dependence as in the case of acoustic polarons, this is only true in the limit $k \ll 1$, and large deviations already occur for $k \approx 1$, the relevant length scale of the problem. Yet even though the transition is not as abrupt as for acoustic polarons, it is possible to distinguish two regimes. In conjunction with the crossover from positive to negative values of the free energy, this is again indicative of a transition between an quasifree (unbound) impurity and self-trapping for the impurity.

\section{Polaron radius}

The second variational parameter, $\Omega$, is linked to the polaron radius. Within the model system described by the action functional $\mathcal{S}_{0}$, expression (13), the expectation value of the square of the relative coordinate for the impurity-boson mass system is given by

$$
\left\langle r^{2}\right\rangle=\frac{3}{2 \Omega} \frac{M}{1+M} \operatorname{coth}\left(\frac{\beta \Omega}{2}\right) .
$$

The square root of this is a measure of the localization length of the impurity wave function. For strong coupling, this expectation value converges to the expectation value with respect to the variational wave function formulated by Landau and Pekar. ${ }^{27}$

Figure 3 shows the polaron radius $\sqrt{\left\langle r^{2}\right\rangle}$ as a function of the coupling strength, for different values of $\beta$, at a cutoff $K_{c}=200$. As the coupling strength is increased, the polaron radius decreases, indicating a stronger confinement of the impurity wave function. In particular the large $\alpha$ behavior might still depend on the cut-off, although a $1 / \alpha$ dependence is expected independently of $K_{c}$. Near $\alpha \approx 3-3.5$, we find that a local maximum develops and the behavior of the po- 


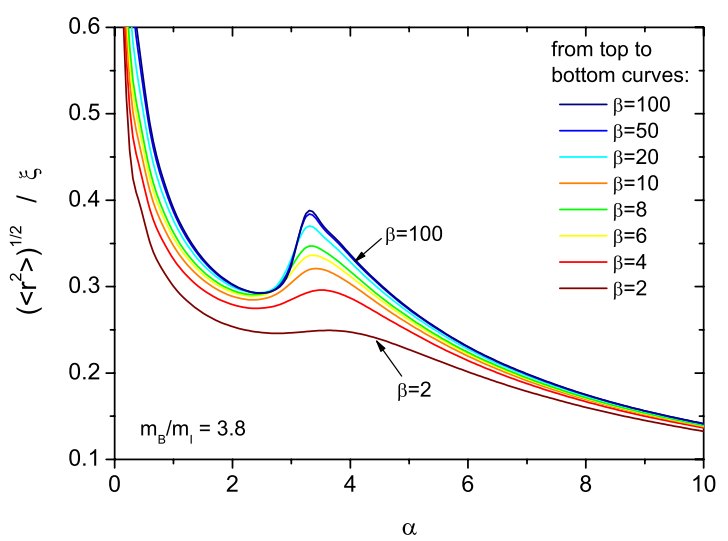

FIG. 3. (Color online) The polaron radius in units of the healing length is plotted as a function of the coupling constant $\alpha$, for different temperatures. From top to bottom, $\beta=100,50,20,10,8,6,4$, and 2 . Around $\alpha \approx 3$ nonmonotonous behavior develops as a function of $\alpha$ when the temperature is reduced.

laron size as a function of $\alpha$ becomes nonmonotonic. It is not clear whether this local maximum is an intrinsic feature of the BEC-impurity polaron, or whether it is an artifact from the particular variational model used.

\section{CONCLUSION}

When the Bogoliubov approximation applies, the Hamiltonian describing the impurity in a condensate can be cast in the form of the Fröhlich polaron Hamiltonian. The physics becomes similar to that of a Fröhlich polaron, where for the impurity in the BEC the Bogoliubov excitations have taken the role of the phonons and the interaction strength is related to the impurity-boson and boson-boson scattering lengths. The most accurate description of polaron physics in the case of electron-phonon interactions is given by Feynman's varia- tional treatment, which moreover allows to study the temperature dependence of observables such as the effective mass and the free energy. We have applied a JensenFeynman path-integral type calculation to the case of the impurity in a condensate and derived expression (18) for the free energy. Both in the free energy and the effective mass, a critical value of the coupling strength $\alpha \approx 3.5$ can be identified where the system crosses over from the weak-coupling to the strong-coupling regime. These regimes show a qualitatively different behavior of the effective mass and free energy. The sharp increase in the effective mass in the strongcoupling regime hints at a transition from an quasifree state to a large-mass state similar to that for acoustic polarons. It has been pointed out ${ }^{13}$ that a transition from the quasifree regime to the large-mass regime is impossible to realize in most semiconductors and III-V compounds, even in alkali halides. However, the present results indicate that it might be attainable in ultracold gases. For this purpose, we investigated the experimentally relevant values of the system parameters, and derived a useful expression (25) relating $\alpha$ to the various parameters in the case of a condensate in the Thomas-Fermi regime. This opens up the prospect to reach and investigate the strong-coupling regime in ultracold gases, whereas this regime has hitherto be inaccessible in the solid state.

\section{ACKNOWLEDGMENTS}

This work was supported by FWO-V under Project Nos. G.0180.09N, G.0115.06, G.0356.06, G.0370.09N, the WOG under Project No. WO.033.09N (Belgium), and INTAS under Project No. 05-104-7656. J.T. gratefully acknowledges support of the Special Research Fund of the University of Antwerp, BOF NOI UA 2004. M.O. acknowledges financial support by the ExtreMe Matter Institute EMMI in the framework of the Helmholtz Alliance under Grant No. HA216/ EMMI.
${ }^{1}$ I. Bloch, J. Dalibard, and W. Zwerger, Rev. Mod. Phys. 80, 885 (2008).

${ }^{2}$ J. T. Devreese and A. S. Alexandrov, Rep. Prog. Phys. 72, 066501 (2009), arXiv:0904.3682.

${ }^{3}$ L. D. Landau and S. I. Pekar, Zh. Eksp. Teor. Fiz. 18, 419 (1948).

${ }^{4}$ H. Fröhlich, Adv. Phys. 3, 325 (1954).

${ }^{5}$ R. P. Feynman, Phys. Rev. 97, 660 (1955).

${ }^{6}$ E. Kartheuser, E. Evrard, and J. Devreese, Phys. Rev. Lett. 22, 94 (1969).

${ }^{7}$ V. L. Gurevich, I. G. Lang, and Yu. A. Firsov, Sov. Phys. Solid State 4, 918 (1962).

${ }^{8}$ J. Devreese, J. De Sitter, and M. Goovaerts, Phys. Rev. B 5, 2367 (1972).

${ }^{9}$ R. Feynman, R. Hellwarth, C. Iddings, and P. Platzman, Phys. Rev. 127, 1004 (1962).

${ }^{10}$ A. S. Mishchenko, N. Nagaosa, N. V. Prokof'ev, A. Sakamoto, and B. V. Svistunov, Phys. Rev. Lett. 91, 236401 (2003).
${ }^{11}$ G. De Filippis, V. Cataudella, A. S. Mishchenko, C. A. Perroni, and J. T. Devreese, Phys. Rev. Lett. 96, 136405 (2006).

${ }^{12}$ G. D. Mahan and J. J. Hopfield, Phys. Rev. Lett. 12, 241 (1964); V. B. Shikin and Y. P. Monarkha, J. Low Temp. Phys. 16, 193 (1974).

${ }^{13}$ F. M. Peeters and J. T. Devreese, Phys. Rev. B 32, 3515 (1985).

${ }^{14}$ A. S. Alexandrov, Phys. Rev. B 77, 094502 (2008); A. S. Alexandrov, Polarons in Advanced Materials, Springer Series in Materials Science (Springer-Verlag, Berlin, 2007), Vol. 103, pp. 257-310, and references therein.

${ }^{15}$ F. M. Cucchietti and E. Timmermans, Phys. Rev. Lett. 96, 210401 (2006).

${ }^{16}$ K. Sacha and E. Timmermans, Phys. Rev. A 73, 063604 (2006).

${ }^{17}$ G. D. Mahan, in Polarons in Ionic Crystals and Polar Semiconductors, edited by J. T. Devreese (North-Holland, Amsterdam, 1972), p. 553.

${ }^{18}$ S. A. Jackson and P. M. Platzman, Phys. Rev. B 24, 499 (1981); G. E. Marques and N. Studart, ibid. 39, 4133 (1989); J. 
Tempere, S. N. Klimin, I. F. Silvera, and J. T. Devreese, Eur. Phys. J. B 32, 329 (2003).

${ }^{19}$ O. Penrose and L. Onsager, Phys. Rev. 104, 576 (1956).

${ }^{20}$ N. N. Bogoliubov, J. Phys. (USSR) 11, 23 (1947).

${ }^{21}$ L. Pitaevskii and S. Stringari, Bose-Einstein Condensation (Oxford Science Publications, Clarendon Press, Oxford, UK, 2003).

${ }^{22}$ B.-B. Huang and S.-L. Wan, Chin. Phys. Lett. 26, 080302 (2009).

${ }^{23}$ M. Bruderer, A. Klein, S. R. Clark, and D. Jaksch, Phys. Rev. A 76, 011605(R) (2007).

${ }^{24}$ R. P. Feynman, Statistical Mechanics: A Set Of Lectures (Addison-Wesley Publ. Co., Reading, MA, 1990).

${ }^{25}$ H. Kleinert, Path Integrals in Quantum Mechanics, Statistics, Polymer Physics, and Financial Markets, 5th ed. (World Scientific, Singapore, 2009).
${ }^{26}$ T. D. Schultz, Phys. Rev. 116, 526 (1959).

${ }^{27}$ L. D. Landau and S. I. Pekar, Zh. Eksp. Teor. Fiz. 16, 341 (1946).

${ }^{28}$ C. Samuelis, E. Tiesinga, T. Laue, M. Elbs, H. Knöckel, and E. Tiemann, Phys. Rev. A 63, 012710 (2000).

${ }^{29}$ M. Gacesa, P. Pellegrini, and R. Côté, Phys. Rev. A 78, 010701(R) (2008).

${ }^{30}$ S. Inouye, M. R. Andrews, J. Stenger, H.-J. Miesner, D. M. Stamper-Kurn, and W. Ketterle, Nature (London) 392, 151 (1998).

${ }^{31}$ C. A. Stan, M. W. Zwierlein, C. H. Schunck, S. M. F. Raupach, and W. Ketterle, Phys. Rev. Lett. 93, 143001 (2004).

${ }^{32}$ A. Derevianko, W. R. Johnson, M. S. Safronova, and J. F. Babb, Phys. Rev. Lett. 82, 3589 (1999). 